\title{
Search for Second-Class Currents in $\tau^{-} \rightarrow \omega \pi^{-} \nu_{\tau}$
}

B. Aubert, ${ }^{1}$ Y. Karyotakis, ${ }^{1}$ J. P. Lees, ${ }^{1}$ V. Poireau, ${ }^{1}$ E. Prencipe,${ }^{1}$ X. Prudent,${ }^{1}$ V. Tisserand, ${ }^{1}$ J. Garra Tico, ${ }^{2}$ E. Grauges, ${ }^{2}$ M. Martinelli, ${ }^{3 a, 3 b}$ A. Palano, ${ }^{3 a, 3 b}$ M. Pappagallo, ${ }^{3 a, 3 b}$ G. Eigen, ${ }^{4}$ B. Stugu, ${ }^{4}$ L. Sun, ${ }^{4}$ M. Battaglia, ${ }^{5}$ D. N. Brown, ${ }^{5}$

L. T. Kerth, ${ }^{5}$ Yu. G. Kolomensky, ${ }^{5}$ G. Lynch, ${ }^{5}$ I. L. Osipenkov, ${ }^{5}$ K. Tackmann, ${ }^{5}$ T. Tanabe, ${ }^{5}$ C. M. Hawkes, ${ }^{6}$ N. Soni, ${ }^{6}$

A. T. Watson, ${ }^{6}$ H. Koch, ${ }^{7}$ T. Schroeder, ${ }^{7}$ D. J. Asgeirsson, ${ }^{8}$ B. G. Fulsom, ${ }^{8}$ C. Hearty, ${ }^{8}$ T. S. Mattison, ${ }^{8}$ J. A. McKenna, ${ }^{8}$

M. Barrett, ${ }^{9}$ A. Khan, ${ }^{9}$ A. Randle-Conde, ${ }^{9}$ V.E. Blinov, ${ }^{10}$ A. D. Bukin,,${ }^{10}$, A. R. Buzykaev,${ }^{10}$ V. P. Druzhinin, ${ }^{10}$

V. B. Golubev, ${ }^{10}$ A. P. Onuchin, ${ }^{10}$ S. I. Serednyakov, ${ }^{10}$ Yu. I. Skovpen, ${ }^{10}$ E. P. Solodov, ${ }^{10}$ K. Yu. Todyshev,${ }^{10}$ M. Bondioli, ${ }^{11}$

S. Curry, ${ }^{11}$ I. Eschrich, ${ }^{11}$ D. Kirkby, ${ }^{11}$ A. J. Lankford, ${ }^{11}$ P. Lund,${ }^{11}$ M. Mandelkern, ${ }^{11}$ E. C. Martin, ${ }^{11}$ D. P. Stoker,${ }^{11}$ H. Atmacan, ${ }^{12}$ J. W. Gary, ${ }^{12}$ F. Liu, ${ }^{12}$ O. Long, ${ }^{12}$ G. M. Vitug, ${ }^{12}$ Z. Yasin, ${ }^{12}$ L. Zhang, ${ }^{12}$ V. Sharma,${ }^{13}$ C. Campagnari, ${ }^{14}$ T. M. Hong, ${ }^{14}$ D. Kovalskyi, ${ }^{14}$ M. A. Mazur, ${ }^{14}$ J. D. Richman, ${ }^{14}$ T. W. Beck, ${ }^{15}$ A. M. Eisner, ${ }^{15}$ C. A. Heusch, ${ }^{15}$

J. Kroseberg, ${ }^{15}$ W. S. Lockman, ${ }^{15}$ A. J. Martinez ${ }^{15}$ T. Schalk, ${ }^{15}$ B. A. Schumm, ${ }^{15}$ A. Seiden, ${ }^{15}$ L. Wang, ${ }^{15}$

L. O. Winstrom, ${ }^{15}$ C. H. Cheng, ${ }^{16}$ D. A. Doll, ${ }^{16}$ B. Echenard,${ }^{16}$ F. Fang, ${ }^{16}$ D. G. Hitlin, ${ }^{16}$ I. Narsky, ${ }^{16}$ T. Piatenko, ${ }^{16}$

F. C. Porter, ${ }^{16}$ R. Andreassen, ${ }^{17}$ G. Mancinelli, ${ }^{17}$ B. T. Meadows,${ }^{17}$ K. Mishra,${ }^{17}$ M. D. Sokoloff, ${ }^{17}$ P. C. Bloom, ${ }^{18}$

W. T. Ford, ${ }^{18}$ A. Gaz, ${ }^{18}$ J. F. Hirschauer, ${ }^{18}$ M. Nagel, ${ }^{18}$ U. Nauenberg, ${ }^{18}$ J. G. Smith, ${ }^{18}$ S. R. Wagner, ${ }^{18}$ R. Ayad, ${ }^{19, \dagger}$

W. H. Toki, ${ }^{19}$ R. J. Wilson, ${ }^{19}$ E. Feltresi, ${ }^{20}$ A. Hauke, ${ }^{20}$ H. Jasper, ${ }^{20}$ T. M. Karbach,${ }^{20}$ J. Merkel, ${ }^{20}$ A. Petzold,${ }^{20}$ B. Spaan,${ }^{20}$

K. Wacker, ${ }^{20}$ M. J. Kobel, ${ }^{21}$ R. Nogowski, ${ }^{21}$ K. R. Schubert, ${ }^{21}$ R. Schwierz, ${ }^{21}$ A. Volk, ${ }^{21}$ D. Bernard, ${ }^{22}$ E. Latour, ${ }^{22}$

M. Verderi, ${ }^{22}$ P. J. Clark, ${ }^{23}$ S. Playfer, ${ }^{23}$ J. E. Watson, ${ }^{23}$ M. Andreotti, ${ }^{24 a, 24 b}$ D. Bettoni, ${ }^{24 a}$ C. Bozzi, ${ }^{24 a}$ R. Calabrese, ${ }^{24 a, 24 b}$ A. Cecchi, ${ }^{24 a, 24 b}$ G. Cibinetto, ${ }^{24 a, 24 b}$ E. Fioravanti, ${ }^{24 a, 24 b}$ P. Franchini ${ }^{24 a, 24 b}$ E. Luppi, ${ }^{24 a, 24 b}$ M. Munerato, ${ }^{24 a, 24 b}$

M. Negrini, ${ }^{24 a, 24 b}$ A. Petrella, ${ }^{24 a, 24 b}$ L. Piemontese, ${ }^{24 a}$ V. Santoro, ${ }^{24 a, 24 b}$ R. Baldini-Ferroli, ${ }^{25}$ A. Calcaterra, ${ }^{25}$

R. de Sangro, ${ }^{25}$ G. Finocchiaro, ${ }^{25}$ S. Pacetti, ${ }^{25}$ P. Patteri, ${ }^{25}$ I. M. Peruzzi, ${ }^{25,}$ M. Piccolo,${ }^{25}$ M. Rama, ${ }^{25}$ A. Zallo, ${ }^{25}$

R. Contri, ${ }^{26 a, 26 b}$ E. Guido, ${ }^{26 a}$ M. Lo Vetere, ${ }^{26 a, 26 b}$ M. R. Monge, ${ }^{26 a, 26 b}$ S. Passaggio, ${ }^{26 a, 26 b}$ C. Patrignani, ${ }^{26 a, 26 b}$

E. Robutti, ${ }^{26 a}$ S. Tosi, ${ }^{26 a, 26 b}$ K. S. Chaisanguanthum, ${ }^{27}$ M. Morii ${ }^{27}$ A. Adametz,${ }^{28}$ J. Marks, ${ }^{28}$ S. Schenk, ${ }^{28}$ U. Uwer, ${ }^{28}$

F. U. Bernlochner, ${ }^{29}$ V. Klose, ${ }^{29}$ H. M. Lacker, ${ }^{29}$ D. J. Bard ${ }^{30}$ P. D. Dauncey, ${ }^{30}$ M. Tibbetts, ${ }^{30}$ P. K. Behera, ${ }^{31}$

M. J. Charles, ${ }^{31}$ U. Mallik, ${ }^{31}$ J. Cochran, ${ }^{32}$ H. B. Crawley, ${ }^{32}$ L. Dong,${ }^{32}$ V. Eyges,${ }^{32}$ W. T. Meyer, ${ }^{32}$ S. Prell, ${ }^{32}$

E. I. Rosenberg, ${ }^{32}$ A. E. Rubin, ${ }^{32}$ Y. Y. Gao, ${ }^{33}$ A. V. Gritsan, ${ }^{33}$ Z. J. Guo, ${ }^{33}$ N. Arnaud,${ }^{34}$ J. Béquilleux,${ }^{34}$ A. D’Orazio, ${ }^{34}$ M. Davier,,${ }^{34}$ D. Derkach, ${ }^{34}$ J. Firmino da Costa,${ }^{34}$ G. Grosdidier, ${ }^{34}$ F. Le Diberder, ${ }^{34}$ V. Lepeltier, ${ }^{34}$ A. M. Lutz, ${ }^{34}$ B. Malaescu, ${ }^{34}$ S. Pruvot,${ }^{34}$ P. Roudeau, ${ }^{34}$ M. H. Schune, ${ }^{34}$ J. Serrano, ${ }^{34}$ V. Sordini, ${ }^{34, \S}$ A. Stocchi, ${ }^{34}$ G. Wormser, ${ }^{34}$ D. J. Lange, ${ }^{35}$ D. M. Wright, ${ }^{35}$ I. Bingham, ${ }^{36}$ J. P. Burke, ${ }^{36}$ C. A. Chavez,${ }^{36}$ J. R. Fry, ${ }^{36}$ E. Gabathuler, ${ }^{36}$ R. Gamet, ${ }^{36}$

D. E. Hutchcroft, ${ }^{36}$ D. J. Payne, ${ }^{36}$ C. Touramanis, ${ }^{36}$ A. J. Bevan, ${ }^{37}$ C. K. Clarke,${ }^{37}$ F. Di Lodovico, ${ }^{37}$ R. Sacco, ${ }^{37}$

M. Sigamani, ${ }^{37}$ G. Cowan, ${ }^{38}$ S. Paramesvaran, ${ }^{38}$ A. C. Wren, ${ }^{38}$ D. N. Brown, ${ }^{39}$ C. L. Davis, ${ }^{39}$ A. G. Denig, ${ }^{40}$ M. Fritsch, ${ }^{40}$ W. Gradl ${ }^{40}$ A. Hafner ${ }^{40}$ K. E. Alwyn, ${ }^{41}$ D. Bailey, ${ }^{41}$ R. J. Barlow, ${ }^{41}$ G. Jackson, ${ }^{41}$ G. D. Lafferty, ${ }^{41}$ T. J. West,${ }^{41}$ J. I. Yi ${ }^{41}$ J. Anderson, ${ }^{42}$ C. Chen, ${ }^{42}$ A. Jawahery, ${ }^{42}$ D. A. Roberts, ${ }^{42}$ G. Simi, ${ }^{42}$ J. M. Tuggle, ${ }^{42}$ C. Dallapiccola, ${ }^{43}$ E. Salvati, ${ }^{43}$ S. Saremi,${ }^{43}$ R. Cowan, ${ }^{44}$ D. Dujmic,${ }^{44}$ P. H. Fisher,${ }^{44}$ S. W. Henderson, ${ }^{44}$ G. Sciolla,${ }^{44}$ M. Spitznagel, ${ }^{44}$ R. K. Yamamoto, ${ }^{44}$

M. Zhao ${ }^{44}$ P. M. Patel, ${ }^{45}$ S. H. Robertson, ${ }^{45}$ M. Schram, ${ }^{45}$ A. Lazzaro, ${ }^{46 a, 46 b}$ V. Lombardo, ${ }^{46 a}$ F. Palombo, ${ }^{46 a, 46 b}$

S. Stracka, ${ }^{46 a, 46 b}$ J. M. Bauer, ${ }^{47}$ L. Cremaldi ${ }^{47}$ R. Godang, ${ }^{47, \|}$ R. Kroeger, ${ }^{47}$ P. Sonnek, ${ }^{47}$ D. J. Summers, ${ }^{47}$ H. W. Zhao, ${ }^{47}$

M. Simard, ${ }^{48}$ P. Taras, ${ }^{48}$ H. Nicholson, ${ }^{49}$ G. De Nardo,${ }^{50 a, 50 b}$ L. Lista, ${ }^{50 a}$ D. Monorchio, ${ }^{50 a, 50 b}$ G. Onorato, ${ }^{50 a, 50 b}$

C. Sciacca, ${ }^{50 a, 50 b}$ G. Raven, ${ }^{51}$ H. L. Snoek, ${ }^{51}$ C. P. Jessop, ${ }^{52}$ K. J. Knoepfel, ${ }^{52}$ J. M. LoSecco, ${ }^{52}$ W. F. Wang, ${ }^{52}$

L. A. Corwin, ${ }^{53}$ K. Honscheid, ${ }^{53}$ H. Kagan,${ }^{53}$ R. Kass, ${ }^{53}$ J. P. Morris, ${ }^{53}$ A. M. Rahimi,${ }^{53}$ J. J. Regensburger, ${ }^{53}$ S. J. Sekula, ${ }^{53}$ Q. K. Wong, ${ }^{53}$ N. L. Blount, ${ }^{54}$ J. Brau, ${ }^{54}$ R. Frey, ${ }^{54}$ O. Igonkina, ${ }^{54}$ J. A. Kolb,${ }^{54}$ M. Lu, ${ }^{54}$ R. Rahmat, ${ }^{54}$ N. B. Sinev, ${ }^{54}$

D. Strom, ${ }^{54}$ J. Strube,${ }^{54}$ E. Torrence, ${ }^{54}$ G. Castelli, ${ }^{55 a, 55 b}$ N. Gagliardi, ${ }^{55 a, 55 b}$ M. Margoni,,${ }^{55 a, 55 b}$ M. Morandin, ${ }^{55 a}$

M. Posocco, ${ }^{55 a}$ M. Rotondo, ${ }^{55 a}$ F. Simonetto, ${ }^{55 a, 55 b}$ R. Stroili, ${ }^{55 a, 55 b}$ C. Voci, ${ }^{55 a, 55 b}$ P. del Amo Sanchez ${ }^{56}$ E. Ben-Haim, ${ }^{56}$

G. R. Bonneaud, ${ }^{56}$ H. Briand, ${ }^{56}$ J. Chauveau, ${ }^{56}$ O. Hamon, ${ }^{56}$ Ph. Leruste, ${ }^{56}$ G. Marchiori, ${ }^{56}$ J. Ocariz, ${ }^{56}$ A. Perez, ${ }^{56}$

J. Prendki, ${ }^{56}$ S. Sitt, ${ }^{56}$ L. Gladney, ${ }^{57}$ M. Biasini, ${ }^{58 a, 58 b}$ E. Manoni, ${ }^{58 a, 58 b}$ C. Angelini, ${ }^{59 a, 59 b}$ G. Batignani, ${ }^{59 a, 59 b}$

S. Bettarini, ${ }^{59 a, 59 b}$ G. Calderini, ${ }^{59 a, 59 b, \pi l}$ M. Carpinelli, ${ }^{59 a, 59 b, * *}$ A. Cervelli, ${ }^{59 a, 59 b}$ F. Forti, ${ }^{59 a, 59 b}$ M. A. Giorgi, ${ }^{59 a, 59 b}$ A. Lusiani, ${ }^{59 a, 59 c}$ M. Morganti, ${ }^{59 a, 59 b}$ N. Neri, ${ }^{59 a, 59 b}$ E. Paoloni, ${ }^{59 a, 59 b}$ G. Rizzo, ${ }^{59 a, 59 b}$ J. J. Walsh, ${ }^{59 a, 59 b}$ D. Lopes Pegna, ${ }^{60}$ C. Lu, ${ }^{60}$ J. Olsen, ${ }^{60}$ A. J. S. Smith,${ }^{60}$ A. V. Telnov ${ }^{60}$ F. Anulli, ${ }^{61 a}$ E. Baracchini, ${ }^{61 a, 61 b}$ G. Cavoto, ${ }^{61 a}$ R. Faccini, ${ }^{61 a, 61 b}$ F. Ferrarotto, ${ }^{61 \mathrm{a}}$ F. Ferroni, ${ }^{61 \mathrm{a}, 61 \mathrm{~b}}$ M. Gaspero, ${ }^{61 \mathrm{a}, 61 \mathrm{~b}}$ P. D. Jackson, ${ }^{61 \mathrm{a}}$ L. Li Gioi, ${ }^{61 \mathrm{a}}$ M. A. Mazzoni, ${ }^{61 \mathrm{a}}$ S. Morganti, ${ }^{61 \mathrm{a}}$ G. Piredda, ${ }^{61 \mathrm{a}}$ F. Renga, ${ }^{61 \mathrm{a}, 61 \mathrm{~b}}$ C. Voena,${ }^{61 \mathrm{a}}$ M. Ebert, ${ }^{62}$ T. Hartmann, ${ }^{62}$ H. Schröder, ${ }^{62}$ R. Waldi, ${ }^{62}$ T. Adye,${ }^{63}$ B. Franek ${ }^{63}$ E. O. Olaiya ${ }^{63}$ F. F. Wilson, ${ }^{63}$ S. Emery, ${ }^{64}$ L. Esteve, ${ }^{64}$ G. Hamel de Monchenault, ${ }^{64}$ W. Kozanecki, ${ }^{64}$ G. Vasseur, ${ }^{64}$ 
Ch. Yèche, ${ }^{64}$ M. Zito, ${ }^{64}$ M. T. Allen ${ }^{65}$ D. Aston,${ }^{65}$ R. Bartoldus, ${ }^{65}$ J. F. Benitez, ${ }^{65}$ R. Cenci, ${ }^{65}$ J. P. Coleman, ${ }^{65}$ M. R. Convery, ${ }^{65}$ J. C. Dingfelder ${ }^{65}$ J. Dorfan, ${ }^{65}$ G. P. Dubois-Felsmann, ${ }^{65}$ W. Dunwoodie, ${ }^{65}$ R. C. Field ${ }^{65}$ M. Franco Sevilla, ${ }^{65}$ A. M. Gabareen, ${ }^{65}$ M. T. Graham, ${ }^{65}$ P. Grenier, ${ }^{65}$ C. Hast, ${ }^{65}$ W. R. Innes, ${ }^{65}$ J. Kaminski, ${ }^{65}$ M. H. Kelsey, ${ }^{65}$ H. Kim, ${ }^{65}$ P. Kim,${ }^{65}$ M. L. Kocian, ${ }^{65}$ D. W. G. S. Leith, ${ }^{65}$ S. Li,${ }^{65}$ B. Lindquist,${ }^{65}$ S. Luitz, ${ }^{65}$ V. Luth, ${ }^{65}$ H. L. Lynch, ${ }^{65}$ D. B. MacFarlane, ${ }^{65}$ H. Marsiske, ${ }^{65}$ R. Messner, ${ }^{65}, *$ D. R. Muller, ${ }^{65}$ H. Neal,${ }^{65}$ S. Nelson, ${ }^{65}$ C. P. O' Grady,${ }^{65}$ I. Ofte, ${ }^{65}$ M. Perl, ${ }^{65}$ B. N. Ratcliff, ${ }^{65}$ A. Roodman, ${ }^{65}$ A. A. Salnikov, ${ }^{65}$ R. H. Schindler,${ }^{65}$ J. Schwiening, ${ }^{65}$ A. Snyder ${ }^{65}$ D. Su, ${ }^{65}$ M. K. Sullivan, ${ }^{65}$ K. Suzuki ${ }^{65}$ S. K. Swain, ${ }^{65}$ J. M. Thompson, ${ }^{65}$ J. Va'vra, ${ }^{65}$ A. P. Wagner ${ }^{65}$ M. Weaver ${ }^{65}$ C. A. West, ${ }^{65}$ W. J. Wisniewski, ${ }^{65}$ M. Wittgen,${ }^{65}$ D. H. Wright, ${ }^{65}$ H. W. Wulsin, ${ }^{65}$ A. K. Yarritu ${ }^{65}$ C. C. Young, ${ }^{65}$ V. Ziegler, ${ }^{65}$ X. R. Chen,${ }^{66}$ H. Liu, ${ }^{66}$ W. Park ${ }^{66}$ M. V. Purohit ${ }^{66}$ R. M. White, ${ }^{66}$ J. R. Wilson, ${ }^{66}$ P. R. Burchat, ${ }^{67}$ A. J. Edwards, ${ }^{67}$ T. S. Miyashita, ${ }^{67}$ S. Ahmed, ${ }^{68}$ M. S. Alam, ${ }^{68}$ J. A. Ernst, ${ }^{68}$ B. Pan, ${ }^{68}$ M. A. Saeed ${ }^{68}$ S. B. Zain, ${ }^{68}$ A. Soffer ${ }^{69}$ S. M. Spanier ${ }^{70}$ B. J. Wogsland,${ }^{70}$ R. Eckmann, ${ }^{71}$ J. L. Ritchie, ${ }^{71}$ A. M. Ruland, ${ }^{71}$ C. J. Schilling, ${ }^{71}$ R. F. Schwitters, ${ }^{71}$ B. C. Wray, ${ }^{71}$ B. W. Drummond, ${ }^{72}$ J. M. Izen, ${ }^{72}$ X. C. Lou, ${ }^{72}$ F. Bianchi, ${ }^{73 a, 73 b}$ D. Gamba, ${ }^{73 a, 73 b}$ M. Pelliccioni, ${ }^{73 a, 73 b}$ M. Bomben, ${ }^{74 a, 74 b}$ L. Bosisio, ${ }^{74 a, 74 b}$ C. Cartaro, ${ }^{74 a, 74 b}$ G. Della Ricca, ${ }^{74 a, 74 b}$ L. Lanceri, ${ }^{74 a, 74 b}$ L. Vitale ${ }^{74 a, 74 b}$ V. Azzolini, ${ }^{75}$ N. Lopez-March, ${ }^{75}$ F. Martinez-Vidal,${ }^{75}$ D. A. Milanes, ${ }^{75}$ A. Oyanguren, ${ }^{75}$ J. Albert, ${ }^{76}$ Sw. Banerjee, ${ }^{76}$ B. Bhuyan, ${ }^{76}$ H. H. F. Choi, ${ }^{76}$ K. Hamano, ${ }^{76}$ G. J. King,${ }^{76}$ R. Kowalewski, ${ }^{76}$ M. J. Lewczuk, ${ }^{76}$ I. M. Nugent, ${ }^{76}$ J. M. Roney, ${ }^{76}$ R. J. Sobie, ${ }^{76}$ T. J. Gershon, ${ }^{77}$ P. F. Harrison, ${ }^{77}$ J. Ilic, ${ }^{77}$ T. E. Latham, ${ }^{77}$ G. B. Mohanty ${ }^{77}$ E. M. T. Puccio, ${ }^{77}$ H. R. Band, ${ }^{78}$ X. Chen, ${ }^{78}$ S. Dasu, ${ }^{78}$ K. T. Flood,${ }^{78}$ Y. Pan,${ }^{78}$ R. Prepost,${ }^{78}$ C. O. Vuosalo, ${ }^{78}$ and S. L. $\mathrm{Wu}^{78}$

(The BABAR Collaboration)

${ }^{1}$ Laboratoire d'Annecy-le-Vieux de Physique des Particules (LAPP), Université de Savoie, CNRS/IN2P3, F-74941 Annecy-Le-Vieux, France

${ }^{2}$ Universitat de Barcelona, Facultat de Fisica, Departament ECM, E-08028 Barcelona, Spain ${ }^{3 a}$ INFN Sezione di Bari, I-70126 Bari, Italy

${ }^{3 \mathrm{~b}}$ Dipartimento di Fisica, Università di Bari, I-70126 Bari, Italy

${ }^{4}$ University of Bergen, Institute of Physics, N-5007 Bergen, Norway

${ }^{5}$ Lawrence Berkeley National Laboratory and University of California, Berkeley, California 94720, USA

${ }^{6}$ University of Birmingham, Birmingham, B15 2TT, United Kingdom

${ }^{7}$ Ruhr Universität Bochum, Institut für Experimentalphysik 1, D-44780 Bochum, Germany

${ }^{8}$ University of British Columbia, Vancouver, British Columbia, Canada V6T IZ1

${ }^{9}$ Brunel University, Uxbridge, Middlesex UB8 3PH, United Kingdom

${ }^{10}$ Budker Institute of Nuclear Physics, Novosibirsk 630090, Russia

${ }^{11}$ University of California at Irvine, Irvine, California 92697, USA

${ }^{12}$ University of California at Riverside, Riverside, California 92521, USA

${ }^{13}$ University of California at San Diego, La Jolla, California 92093, USA

${ }^{14}$ University of California at Santa Barbara, Santa Barbara, California 93106, USA

${ }^{15}$ University of California at Santa Cruz, Institute for Particle Physics, Santa Cruz, California 95064, USA

${ }^{16}$ California Institute of Technology, Pasadena, California 91125, USA

${ }^{17}$ University of Cincinnati, Cincinnati, Ohio 45221, USA

${ }^{18}$ University of Colorado, Boulder, Colorado 80309, USA

${ }^{19}$ Colorado State University, Fort Collins, Colorado 80523, USA

${ }^{20}$ Technische Universität Dortmund, Fakultät Physik, D-44221 Dortmund, Germany

${ }^{21}$ Technische Universität Dresden, Institut für Kern- und Teilchenphysik, D-01062 Dresden, Germany

${ }^{22}$ Laboratoire Leprince-Ringuet, CNRS/IN2P3, Ecole Polytechnique, F-91128 Palaiseau, France

${ }^{23}$ University of Edinburgh, Edinburgh EH9 3JZ, United Kingdom

${ }^{24 a}$ INFN Sezione di Ferrara, I-44100 Ferrara, Italy

${ }^{24 \mathrm{~b}}$ Dipartimento di Fisica, Università di Ferrara, I-44100 Ferrara, Italy

${ }^{25}$ INFN Laboratori Nazionali di Frascati, I-00044 Frascati, Italy

${ }^{26 a}$ INFN Sezione di Genova, I-16146 Genova, Italy

${ }^{26 \mathrm{~b}}$ Dipartimento di Fisica, Università di Genova, I-16146 Genova, Italy

${ }^{27}$ Harvard University, Cambridge, Massachusetts 02138, USA

${ }^{28}$ Universität Heidelberg, Physikalisches Institut, Philosophenweg 12, D-69120 Heidelberg, Germany

${ }^{29}$ Humboldt-Universität zu Berlin, Institut für Physik, Newtonstr. 15, D-12489 Berlin, Germany

${ }^{30}$ Imperial College London, London, SW7 2AZ, United Kingdom

${ }^{31}$ University of Iowa, Iowa City, Iowa 52242, USA

${ }^{32}$ Iowa State University, Ames, Iowa 50011-3160, USA 


\author{
${ }^{33}$ Johns Hopkins University, Baltimore, Maryland 21218, USA \\ ${ }^{34}$ Laboratoire de l'Accélérateur Linéaire, IN2P3/CNRS et Université Paris-Sud 11, Centre Scientifique d'Orsay, \\ B. P. 34, F-91898 Orsay Cedex, France \\ ${ }^{35}$ Lawrence Livermore National Laboratory, Livermore, California 94550, USA \\ ${ }^{36}$ University of Liverpool, Liverpool L69 7ZE, United Kingdom \\ ${ }^{37}$ Queen Mary, University of London, London, E1 4NS, United Kingdom \\ ${ }^{38}$ University of London, Royal Holloway and Bedford New College, Egham, Surrey TW20 OEX, United Kingdom \\ ${ }^{39}$ University of Louisville, Louisville, Kentucky 40292, USA \\ ${ }^{40}$ Johannes Gutenberg-Universität Mainz, Institut für Kernphysik, D-55099 Mainz, Germany \\ ${ }^{41}$ University of Manchester, Manchester M13 9PL, United Kingdom \\ ${ }^{42}$ University of Maryland, College Park, Maryland 20742, USA \\ ${ }^{43}$ University of Massachusetts, Amherst, Massachusetts 01003, USA \\ ${ }^{44}$ Massachusetts Institute of Technology, Laboratory for Nuclear Science, Cambridge, Massachusetts 02139, USA \\ ${ }^{45}$ McGill University, Montréal, Québec, Canada H3A $2 T 8$ \\ ${ }^{46 a}$ INFN Sezione di Milano, I-20133 Milano, Italy \\ ${ }^{46 \mathrm{~b}}$ Dipartimento di Fisica, Università di Milano, I-20133 Milano, Italy \\ ${ }^{47}$ University of Mississippi, University, Mississippi 38677, USA \\ ${ }^{48}$ Université de Montréal, Physique des Particules, Montréal, Québec, Canada H3C 3J7 \\ ${ }^{49}$ Mount Holyoke College, South Hadley, Massachusetts 01075, USA \\ 50a INFN Sezione di Napoli, I-80126 Napoli, Italy \\ ${ }^{50 \mathrm{~b}}$ Dipartimento di Scienze Fisiche, Università di Napoli Federico II, I-80126 Napoli, Italy \\ ${ }^{51}$ NIKHEF, National Institute for Nuclear Physics and High Energy Physics, NL-1009 DB Amsterdam, The Netherlands \\ ${ }^{52}$ University of Notre Dame, Notre Dame, Indiana 46556, USA \\ ${ }^{53}$ Ohio State University, Columbus, Ohio 43210, USA \\ ${ }^{54}$ University of Oregon, Eugene, Oregon 97403, USA \\ ${ }^{55}$ INFN Sezione di Padova, I-35131 Padova, Italy \\ ${ }^{55 b}$ Dipartimento di Fisica, Università di Padova, I-35131 Padova, Italy \\ ${ }^{56}$ Laboratoire de Physique Nucléaire et de Hautes Energies, IN2P3/CNRS, Université Pierre et Marie Curie-Paris6, \\ Université Denis Diderot-Paris7, F-75252 Paris, France \\ ${ }^{57}$ University of Pennsylvania, Philadelphia, Pennsylvania 19104, USA \\ ${ }^{58}$ INFN Sezione di Perugia, I-06100 Perugia, Italy \\ ${ }^{58 \mathrm{~b}}$ Dipartimento di Fisica, Università di Perugia, I-06100 Perugia, Italy \\ ${ }^{59 a}$ INFN Sezione di Pisa, I-56127 Pisa, Italy \\ ${ }^{59 b}$ Dipartimento di Fisica, Università di Pisa, I-56127 Pisa, Italy \\ ${ }^{59 \mathrm{c}}$ Scuola Normale Superiore di Pisa, I-56127 Pisa, Italy \\ ${ }^{60}$ Princeton University, Princeton, New Jersey 08544, USA \\ ${ }^{61 a}$ INFN Sezione di Roma, I-00185 Roma, Italy \\ ${ }^{61 \mathrm{~b}}$ Dipartimento di Fisica, Università di Roma La Sapienza, I-00185 Roma, Italy \\ ${ }^{62}$ Universität Rostock, D-18051 Rostock, Germany \\ ${ }^{63}$ Rutherford Appleton Laboratory, Chilton, Didcot, Oxon, OX11 0QX, United Kingdom \\ ${ }^{64}$ CEA, Irfu, SPP, Centre de Saclay, F-91191 Gif-sur-Yvette, France \\ ${ }^{65}$ SLAC National Accelerator Laboratory, Stanford, California 94309 USA \\ ${ }^{66}$ University of South Carolina, Columbia, South Carolina 29208, USA \\ ${ }^{67}$ Stanford University, Stanford, California 94305-4060, USA \\ ${ }^{68}$ State University of New York, Albany, New York 12222, USA \\ ${ }^{69}$ Tel Aviv University, School of Physics and Astronomy, Tel Aviv, 69978, Israel \\ ${ }^{70}$ University of Tennessee, Knoxville, Tennessee 37996, USA \\ ${ }^{71}$ University of Texas at Austin, Austin, Texas 78712, USA \\ ${ }^{72}$ University of Texas at Dallas, Richardson, Texas 75083, USA \\ ${ }^{73 a}$ INFN Sezione di Torino, I-10125 Torino, Italy \\ ${ }^{73 b}$ Dipartimento di Fisica Sperimentale, Università di Torino, I-10125 Torino, Italy \\ ${ }^{74 \mathrm{a}}$ INFN Sezione di Trieste, I-34127 Trieste, Italy \\ ${ }^{74 b}$ Dipartimento di Fisica, Università di Trieste, I-34127 Trieste, Italy \\ ${ }^{75}$ IFIC, Universitat de Valencia-CSIC, E-46071 Valencia, Spain \\ ${ }^{76}$ University of Victoria, Victoria, British Columbia, Canada V8W $3 P 6$ \\ ${ }^{77}$ Department of Physics, University of Warwick, Coventry CV4 7AL, United Kingdom \\ ${ }^{78}$ University of Wisconsin, Madison, Wisconsin 53706, USA
}

(Received 20 April 2009; published 22 July 2009)

We report an analysis of $\tau^{-}$decaying into $\omega \pi^{-} \nu_{\tau}$ with $\omega \rightarrow \pi^{+} \pi^{-} \pi^{0}$ using a data sample containing nearly $320 \times 10^{6} \tau$ pairs collected with the $B A B A R$ detector at the PEP-II $B$-Factory. We find no evidence 
for second-class currents, and we set an upper limit of $0.69 \%$ at $90 \%$ confidence level for the fraction of second-class currents in this decay mode.

Hadronic weak currents can be classified as either firstor second-class depending on the spin $J$, parity $P$, and $G$-parity $G$ of the final hadronic system [1]. In the Standard Model, first-class currents (FCC) in $\tau$ decays have $J^{P G}=0^{++}, 0^{--}, 1^{+-}$, or $1^{-+}$, and are expected to dominate. Second-class currents (SCC) have $J^{P G}=0^{+-}$, $0^{-+}, 1^{++}$, or $1^{--}$, and are associated with a decay constant proportional to the mass difference between up and down quarks. Thus, they are expected to vanish in the limit of perfect isospin symmetry. A number of mechanisms have been suggested [2], including possible exchange of Higgs bosons, that could lead to an enhanced rate for secondclass currents. Searches have taken place extensively in nuclear $\beta$ decay experiments $[3,4]$, with no confirmed observations. This Letter presents a search for SCC in $\tau^{-} \rightarrow \omega \pi^{-} \nu_{\tau}$ decays with $\omega \rightarrow \pi^{+} \pi^{-} \pi^{0}$, based on studying the angular distributions of final-state particles.

The decay $\tau^{-} \rightarrow \omega \pi^{-} \nu_{\tau}$ [5] is expected to proceed predominantly through FCC mediated by the $\rho$ resonance. This decay may also potentially proceed through $\mathrm{SCC}$ with $J^{P G}=0^{-+}$or $1^{++}$. The latter may be mediated by $b_{1}(1235)$ [6] with $\tau^{-} \rightarrow b_{1}^{-} \nu_{\tau} \rightarrow \omega \pi^{-} \nu_{\tau}$. The decay $b_{1}^{-} \rightarrow \omega \pi^{-}$occurs through $S$ - and $D$ waves [7], as compared to a $P$ wave for FCC. Different alignments of $\omega$ spin result in different angular distributions of the final-state particles. The expected distributions of $\cos \theta_{\omega \pi}$, $F\left(\cos \theta_{\omega \pi}\right)$, for all possible spin-parity states of the finalstate particles are listed in Table I, where $\theta_{\omega \pi}$ is the angle between the normal to the $\omega$ decay plane and the direction of the remaining $\pi$ in the $\omega$ rest frame. The existing measurements of the angular distribution in $\tau^{-} \rightarrow \omega \pi^{-} \nu_{\tau}$ are consistent with having only the $P$-wave contribution, and the present upper limit is $5.4 \%$ for the ratio of SCC to FCC contributions at $90 \%$ confidence level (CL) $[8,9]$.

This analysis is based on data recorded by the BABAR detector [10] at the PEP-II asymmetric-energy $e^{+} e^{-}$storage rings operated at the SLAC National Accelerator Laboratory. The data sample consists of $347.3 \mathrm{fb}^{-1}$ recorded at a center-of-mass energy of $10.58 \mathrm{GeV}$. With a cross section for $\tau$ pairs of $\sigma_{\tau \tau}=(0.919 \pm 0.003) \mathrm{nb}$ $[11,12]$, this data sample contains nearly $320 \times 10^{6}$ pairs of $\tau$ decays.

The BABAR detector is described in detail in Ref. [10]. Charged-particle tracks are measured with a 5-layer double-sided silicon vertex tracker (SVT) together with a 40-layer drift chamber (DCH) inside a 1.5-T superconducting solenoid magnet. An electromagnetic calorimeter (EMC) consisting of $6580 \mathrm{CsI}(\mathrm{Tl})$ crystals is used for identification of electrons and photons. Charged hadrons are identified by a ring-imaging Cherenkov detector in combination with energy-loss measurements $(d E / d x)$ in the SVT and the DCH. An instrumented magnetic-flux return (IFR) provides muon identification.

Monte Carlo simulation is used to estimate the signal efficiencies and background contamination. KK2F [12] is used to generate $\tau$ pairs with the decays of the $\tau$ leptons modeled by TAUOLA [13]. Continuum $q \bar{q}$ events are simulated using JETSET [14]. Final-state radiative effects are generated for all decays using PHOTOS [15]. The detector response is simulated with GEANT4 [16], and the Monte Carlo events are reconstructed in the same manner as data.

Since $\tau$ pairs are produced back to back in the $e^{+} e^{-}$ center-of-mass frame, each event is divided into two hemispheres according to the thrust axis [17], calculated using all reconstructed charged particles. Candidate events in this analysis are required to have a "1-3 topology," where one track is in one hemisphere (tag hemisphere) and three tracks are in the other hemisphere (signal hemisphere). Events with four well-reconstructed tracks and zero net charge are selected for further analysis. The polar angles of all four tracks and the neutral clusters used in $\pi^{0}$ reconstruction are required to be within the calorimeter acceptance range. Events are rejected if the invariant mass of any pair of oppositely charged tracks, assuming electron mass hypotheses, is less than $90 \mathrm{MeV} / c^{2}$, as these tracks are likely to be from photon conversions in the detector material.

The charged particle found in the tag hemisphere must be either an electron or a muon candidate. Electrons are identified using the ratio of calorimeter energy to track momentum $(E / p)$, the shape of the shower in the calorimeter, and $d E / d x$. Muons are identified by signals in the IFR and small energy deposits in the calorimeter consistent with expectation for a minimum-ionizing particle. Charged particles found in the signal hemisphere must be identified as pion candidates. The $\pi^{0}$ candidates are reconstructed from two separate EMC clusters with energies above $100 \mathrm{MeV}$ that are not associated with charged tracks; these $\pi^{0}$ candidates are required to have invariant masses be-

TABLE I. Expected angular distributions, $F\left(\cos \theta_{\omega \pi}\right)$, for possible spin-parity states in the decay $\tau^{-} \rightarrow \omega \pi^{-} \nu_{\tau}$. $L$ is the orbital angular momentum.

\begin{tabular}{lcc}
\hline \hline$J^{P}$ & $L$ & $F\left(\cos \theta_{\omega \pi}\right)$ \\
\hline $1^{-}$ & 1 & $\left(1-\cos ^{2} \theta_{\omega \pi}\right)$ \\
$0^{-}$ & 1 & $\cos ^{2} \theta_{\omega \pi}$ \\
$1^{+}$ & 0 & 1 \\
$1^{+}$ & 2 & $\left(1+3 \cos ^{2} \theta_{\omega \pi}\right)$ \\
\hline \hline
\end{tabular}


tween 100 and $160 \mathrm{MeV} / c^{2}$. Events are required to have a single $\pi^{0}$ in the signal hemisphere. The $\tau$ candidates are reconstructed in the signal hemisphere using the three tracks and the $\pi^{0}$ candidate. The invariant mass of the $\tau$ candidate, $m\left(\pi^{-} \pi^{-} \pi^{+} \pi^{0}\right)$, is required to be less than the mass of the $\tau$ lepton. The Monte Carlo simulation predicts that $14 \%$ of the events remaining after the event selection process are $\tau$-pair events that do not contain a $\tau^{-} \rightarrow$ $\pi^{-} \pi^{-} \pi^{+} \pi^{0} \nu_{\tau}$ decay, and $1.3 \%$ are $e^{+} e^{-} \rightarrow q \bar{q}$ events.

Each selected event has two $\pi^{+} \pi^{-} \pi^{0}$ combinations. The $\omega$ signal region is defined for masses $m\left(\pi^{+} \pi^{-} \pi^{0}\right)$ between $760 \mathrm{MeV} / c^{2}$ and $800 \mathrm{MeV} / c^{2}$ with mass regions of width $60 \mathrm{MeV} / c^{2}$ on each side of the peak used as sideband regions for background studies, as shown in Fig. 1. For each $\omega$ candidate, the angle $\theta_{\omega \pi}$ is calculated. The distribution of $\cos \theta_{\omega \pi}$ is used for the SCC measurement.

There are three background sources to be considered in this analysis: combinatoric background, $q \bar{q}$ events, and nonsignal $\tau$ decays. The combinatoric background is expected, and confirmed by the simulation, to have a distribution of $\cos \theta_{\omega \pi}$ which is independent of $m\left(\pi^{+} \pi^{-} \pi^{0}\right)$. This allows the sideband regions to be used to subtract this background. The number of combinatoric events lying within the signal region is obtained by fitting the $m\left(\pi^{+} \pi^{-} \pi^{0}\right)$ spectrum with a relativistic Breit-Wigner convolved with a resolution function for the $\omega$ resonance and a polynomial for the combinatoric background. The polynomial is integrated over the signal region to find the number of continuum events in the signal region.

After subtracting the combinatoric background, approximately $0.3 \%$ of the remaining events in the signal region are expected to be of $q \bar{q}$ origin, while $4.6 \%$ are expected to be from nonsignal $\tau$ decays. The background from $e^{+} e^{-} \rightarrow q \bar{q}$ events that contain $\omega \rightarrow \pi^{+} \pi^{-} \pi^{0}$ de-

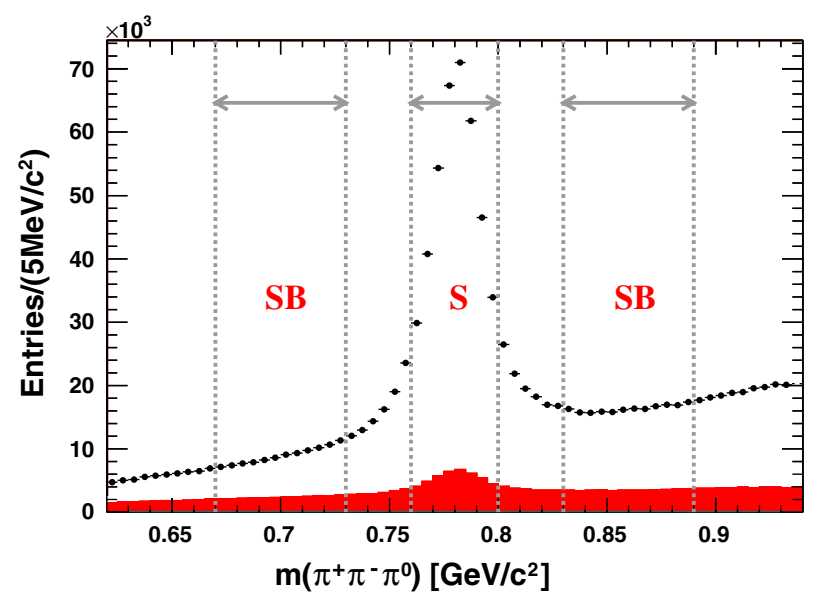

FIG. 1 (color online). $\omega$ candidate mass spectra for selected events in data and background expected from simulation (shaded histogram). The background histogram does not include the nonresonant $\tau^{-} \rightarrow \pi^{-} \pi^{-} \pi^{+} \pi^{0} \nu_{\tau}$ decays. The signal (S) and sideband $(\mathrm{SB})$ regions are indicated in the figure. cays is studied using events with $m\left(\pi^{-} \pi^{-} \pi^{+} \pi^{0}\right)$ well above the $\tau$ mass $\left(>2.1 \mathrm{GeV} / c^{2}\right)$. In this region, where all events are considered to be of $q \bar{q}$ origin, a comparison of the numbers of $\omega$ mesons in simulation and data is used to scale the simulated $q \bar{q}$ background before subtracting from data.

The dominant nonsignal $\tau$ background, comprising 99\% of the remaining background, is $\tau^{-} \rightarrow \omega \pi^{-} \pi^{0} \nu_{\tau}$, where the additional $\pi^{0}$ has not been reconstructed. The decay $\tau^{-} \rightarrow \omega \pi^{-} \pi^{0} \nu_{\tau}$ has not been well measured and is incorrectly modeled in the Monte Carlo simulation. To correct for the differences between data and Monte Carlo simulations, events with an additional $\pi^{0}$ candidate in the signal hemisphere are selected, using the same cuts discussed above. Using these events, the Monte Carlo branching fraction of $\tau^{-} \rightarrow \omega \pi^{-} \pi^{0} \nu_{\tau}$ is corrected by comparing the numbers of fitted $\omega$ candidates in data and Monte Carlo simulations. The fit function used for this is a relativistic Breit-Wigner convolved with a resolution function plus a polynomial background. The branching fraction obtained using this correction technique is found to be consistent with existing measurements [7]. To correct the angular distribution of $\tau^{-} \rightarrow \omega \pi^{-} \pi^{0} \nu_{\tau}$, backgrounds, consisting of combinatorics, $q \bar{q}$ events, and $\tau^{-} \rightarrow$ $\omega \pi^{-} \nu_{\tau}$ decays (assuming the angular distribution corresponding to the dominant FCC contribution), are subtracted from the $\omega \pi^{-} \pi^{0}$ data sample, and the remaining $\cos \theta_{\omega \pi}$ distribution is used to correct the $\tau^{-} \rightarrow \omega \pi^{-} \pi^{0} \nu_{\tau}$ distribution in the Monte Carlo simulation.

After subtracting backgrounds and applying $\cos \theta_{\omega \pi}$-dependent efficiency corrections, a binned fit to the remaining $\cos \theta_{\omega \pi}$ distribution is carried out using

$$
F\left(\cos \theta_{\omega \pi}\right)=N\left[\frac{1}{2} \epsilon+\frac{3}{4}(1-\epsilon)\left(1-\cos ^{2} \theta_{\omega \pi}\right)\right]
$$

where $N$ is a normalization factor and the parameter $\epsilon$ is the fraction of $\tau^{-} \rightarrow \omega \pi^{-} \nu_{\tau}$ decays that proceed through SCC. In Eq. (1), only the $L=0$ term is used to describe the SCC contribution since this function gives the most conservative estimate of $\epsilon$ (i.e., the largest upper limit).

To help validate the analysis method, the procedures were applied to Monte Carlo samples generated to include small fractions (1\% and $2 \%)$ of the second-class current process $\tau^{-} \rightarrow b_{1}(1235)^{-} \nu_{\tau} \rightarrow \omega \pi^{-} \nu_{\tau}$, as well as to a sample containing no SCC contribution. In each case, the fits to the simulated detector-level, background-subtracted angular distributions returned values consistent with the input fractions of SCC.

The largest contributions to systematic uncertainties on $\epsilon$ are scaling and modeling the Monte Carlo background. The correction applied to the branching fraction of $\tau^{-} \rightarrow$ $\omega \pi^{-} \pi^{0} \nu_{\tau}$ has an error associated with it, determined by the available statistics. This correction factor is adjusted by $\pm 1 \sigma$ to obtain the uncertainty in $\epsilon$ while the errors associated with correcting the angular distribution are folded 
TABLE II. Summary of systematic uncertainties on $\epsilon$.

\begin{tabular}{lc}
\hline \hline Source & Uncertainty $\left(\sigma_{\epsilon}\right)$ \\
\hline $\mathcal{B}\left(\tau^{-} \rightarrow \omega \pi^{-} \pi^{0} \nu_{\tau}\right)$ & $\pm 0.07 \%$ \\
nonsimulated $\tau$ decays & $+0.00 \%$ \\
$q \bar{q}$ scaling & $\pm 0.55 \%$ \\
Total & $\pm 0.01 \%$ \\
\hline \hline
\end{tabular}

into the statistical uncertainty. In addition, there are $\tau$ decays that may be present in the final event sample but which are not included in the simulation. The largest of these are expected to be $\tau^{-} \rightarrow \omega K^{-} \nu_{\tau}, \quad \tau^{-} \rightarrow$ $\omega \pi^{-} \pi^{0} \pi^{0} \nu_{\tau}$, and $\tau^{-} \rightarrow \omega \pi^{-} \pi^{-} \pi^{+} \nu_{\tau}$ decays which, when combined, can add up to $0.2 \%$ of the final event sample. Since the effect that these decays have on the angular distribution is unknown, the extreme cases are taken to obtain the uncertainty. These cases correspond to the decays having either entirely $1-\cos ^{2} \theta_{\omega \pi}$ or entirely $\cos ^{2} \theta_{\omega \pi}$ distributions. The scaling of $q \bar{q}$ events can also affect the measurement of $\epsilon$, and the uncertainty is obtained by adjusting the scaling factor by $\pm 1 \sigma$. These systematic uncertainties are summarized in Table II.

Subsets of the generic Monte Carlo dataset are used in the background studies and in the determination of the efficiencies. Therefore, to estimate the sensitivity of the analysis without the effect of statistical correlations in the Monte Carlo samples, an ensemble of simulated experiments is used. In this study, angular distributions are generated for the signal and sideband regions to simulate the statistics available in the data and various Monte Carlo samples used in the analysis, with $\epsilon=0$ in the signal Monte Carlo simulation. After subtracting background samples, the angular distribution is corrected for efficiency and fitted using Eq. (1). The statistical uncertainty on $\epsilon$ obtained from the fit is $0.63 \%$, which combined with the systematic uncertainties leads to an estimated uncertainty of $\sigma_{\epsilon}={ }_{-0.84}^{+0.64} \%$.

The angular distribution of the final-state particles in data is obtained by subtracting estimated backgrounds as described above. The remaining distribution is corrected for efficiency and fitted using Eq. (1) as shown in Fig. 2. The fit has $\chi^{2} /($ degrees of freedom $)=15.4 / 18$, and the fitted value of $\epsilon$ in the data is $[-0.55 \pm$ 0.58 (stat $\left.{ }_{-0.55}^{+0.08}(\mathrm{syst})\right] \%$, which is consistent with no SCC contribution to $\tau^{-} \rightarrow \omega \pi^{-} \nu_{\tau}$ decays.

The upper limit on $\epsilon$ is obtained using a Bayesian approach [18] with a prior that is flat for $\epsilon>0$ and zero for $\epsilon<0$. The probability distribution for the value of the SCC contribution is a Gaussian with mean $\epsilon=-0.55 \%$ and errors $\sigma_{\epsilon}={ }_{-0.84}^{+0.64} \%$, taken from the simulation studies; however, since negative values of $\epsilon$ are nonphysical, only the positive portion of this probability distribution is used in the limit calculation. The limits obtained from this

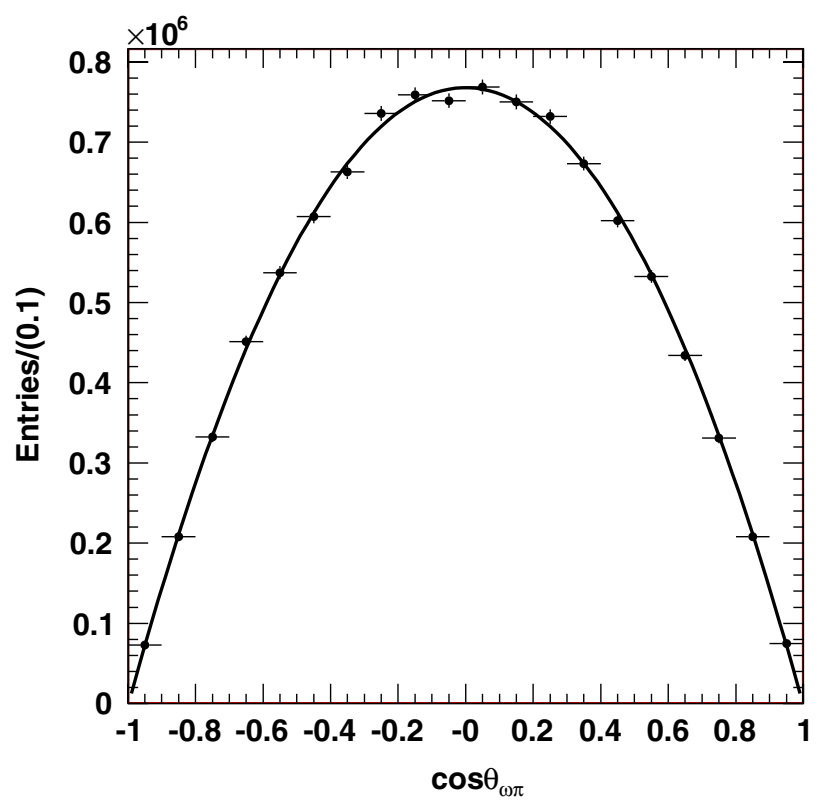

FIG. 2 (color online). The $\cos \theta_{\omega \pi}$ distribution for the data. The curve is the result of the fit described in the text.

method are $\epsilon<0.68 \%$ at $90 \% \mathrm{CL}$ and $\epsilon<0.84 \%$ at 95\% CL.

In summary, a search for second-class currents in the decay $\tau^{-} \rightarrow \omega \pi^{-} \nu_{\tau}$ has been conducted with the BABAR detector. No evidence for second-class currents is observed, and a $90 \%$ confidence level Bayesian upper limit for the fraction of the second-class current in $\tau^{-} \rightarrow$ $\omega \pi^{-} \nu_{\tau}$ decays is set at $0.68 \%$. For comparison with the previous result from CLEO, this is equivalent to a ratio of second-class (nonvector) to first-class (vector) currents of $0.69 \%$. This limit is an order of magnitude lower than the limit set by the CLEO collaboration [9].

We are grateful for the excellent luminosity and machine conditions provided by our PEP-II colleagues, and for the substantial dedicated effort from the computing organizations that support $B A B A R$. The collaborating institutions wish to thank SLAC for its support and kind hospitality. This work is supported by DOE and NSF (USA), NSERC (Canada), CEA and CNRS-IN2P3 (France), BMBF and DFG (Germany), INFN (Italy), FOM (The Netherlands), NFR (Norway), MES (Russia), MEC (Spain), and STFC (United Kingdom). Individuals have received support from the Marie Curie EIF (European Union), and the A. P. Sloan Foundation.

\footnotetext{
* Deceased

${ }^{\dagger}$ Now at Temple University, Philadelphia, PA 19122, USA

${ }^{\star}$ Also with Università di Perugia, Dipartimento di Fisica, Perugia, Italy

${ }^{\S}$ Also with Università di Roma La Sapienza, I-00185 Roma, Italy
} 
${ }^{\|}$Now at University of South Alabama, Mobile, AL 36688, USA

"Also with Laboratoire de Physique Nucléaire et de Hautes Energies, IN2P3/CNRS, Université Pierre et Marie CurieParis6, Université Denis Diderot-Paris7, F-75252 Paris, France

**Also with Università di Sassari, Sassari, Italy

[1] S. Weinberg, Phys. Rev. 112, 1375 (1958).

[2] P. Langacker, Phys. Rev. D 15, 2386 (1977).

[3] T. Sumikama et al., Phys. Lett. B 664, 235 (2008).

[4] K. Minamisono et al., Phys. Rev. C 65, 015501 (2001).

[5] Charge-conjugate reactions are implied throughout this Letter.

[6] C. Leroy and J. Pestieau, Phys. Lett. B 72, 398 (1978).

[7] C. Amsler et al. (Particle Data Group), Phys. Lett. B 667, 1 (2008).

[8] D. Buskulic et al. (ALEPH Collaboration), Z. Phys. C 74, 263 (1997).
[9] K. W. Edwards et al. (CLEO Collaboration), Phys. Rev. D 61, 072003 (2000).

[10] B. Aubert et al. (BABAR Collaboration), Nucl. Instrum. Methods Phys. Res., Sect. A 479, 1 (2002).

[11] S. Banerjee, B. Pietrzyk, J. M. Roney, and Z. Was, Phys. Rev. D 77, 054012 (2008).

[12] S. Jadach, B. F. L. Ward, and Z. Was, Comput. Phys. Commun. 130, 260 (2000).

[13] S. Jadach, Z. Was, R. Decker, and J.H. Kuhn, Comput. Phys. Commun. 76, 361 (1993).

[14] T. Sjostrand, Comput. Phys. Commun. 82, 74 (1994).

[15] E. Barberio and Z. Was, Comput. Phys. Commun. 79, 291 (1994).

[16] S. Agostinelli et al. (GEANT4 Collaboration), Nucl. Instrum. Methods Phys. Res., Sect. A 506, 250 (2003).

[17] S. Brandt et al., Phys. Lett. 12, 57 (1964); E. Farhi, Phys. Rev. Lett. 39, 1587 (1977).

[18] G. J. Feldman and R. D. Cousins, Phys. Rev. D 57, 3873 (1998); R. Barlow, Statistics (Wiley, New York, 1989). 